\section{$1 \mathrm{P253}$}

\section{Modeling of F-actin}

OToshiro Oda ${ }^{1,2}$, Yuichiro Maeda ${ }^{2,3}$

${ }^{1}$ RIKEN Spring-8 center, RIKEN harima institute, RIKEN , ${ }^{2}$ ERATO Actin

Filament Dynamics Project, JST, ${ }^{3}$ School Biological Sciences, Nagoya Univ

To understand the skeletal muscle contraction, we need the structure of myosin head and F-actin. We know the crystal structures of myosin heads bound several kinds of nucleotides. On the other hand, we have not obtained the precise atomic filamentous structure. Last year, we proposed new F-actin structural model using the x-ray fiber diffraction data up to ca $8 \mathrm{~A}$ but we cannot precisely decide the form of the DNase I binding loop and the hydrophobic loop. In this study, we used diffraction data up to $3 \mathrm{~A}$ to increased confirmation of our model. First we calculated the elastic normal vibration modes of actin. Using the liner combination of the lowest 12 mode motions, we searched the conformation that account for diffraction patterns up to $7.2 \mathrm{~A}$. When the search was done form initial conformation of open-crystal structure and close-crystal structure, the resulting conformations were converged into the similar tilted conformation. Secondly, to refine the structure, we carried out the molecular dynamics simulation with a reflection term using FX-plor. MD calculation against 56-6.5 A reflection data and MD calculation against 5.5-3.3 A reflection data was repeatedly done and the temperature was gradually decreased form 400 to $0 \mathrm{~K}$. For the resulting structures, we calculated R-factor against 6.5-5.5 A reflection and we judged the reasonable model using the R-factor against the non-fitting area. We found a part of the DNase I binding loop was present between two long-pitch strands and that the hydrophobic plug was partly extended.

\section{$1 P 255$}

Fiber diffraction study on flagellar axonemes from a wild type and mutant strains of Chlamydomonas

O Shiori Toba ${ }^{1}$, Hiroyuki Iwamoto ${ }^{2}$, Tetsuro Fujisawa ${ }^{3}$, Hitoshi Sakakibara', Kazuhiro Oiwa ${ }^{1}$

'Kobe Advanced ICT Research Center, National Institute of Information and Communications Technology, ${ }^{2}$ Research and Utilization Division, Japan Synchrotron Radiation Research Institute, SPring- $8,{ }^{3}$ Biometal Science Laboratory, RIKEN Harima Institute

Bend formation and propagation of flagella require the precise control of coordination among structural components in an axoneme. To elucidate this mechanism, organization of axonemal components in situ was studied using fiber diffractions of whole flagella isolated from several strains of Chlamydomonas: wild type, outer arm-less mutant (odal), spoke-less mutant ( $p f 14)$, central apparatus-less mutant (pfl8) and $\alpha$ outer arm dynein-less mutant (odall). We aligned axonemes in a flow cell by continuous shear flow, then obtained diffraction patterns with the synchrotron radiation $\mathrm{x}$-ray of $0.9 \AA$ wavelength at SPring $8, \mathrm{BL} 45-\mathrm{XU}$. The diffraction pattern showed several distinct meridional and equatorial reflections. The 24-nm meridional reflection of the wild type was stronger in the presence of ADP-vanadate than odal, and was derived mainly from the structural repeat of the outer dynein arms. The weaker 32-nm meridional reflection of pfl4 than the wild type mainly reflects the structural repeat of the spoke. The 19-nm equatorial reflection of $p f 18$ weaker than the wild type was presumably caused by the lack of the central sheath in pf18. Diffraction patterns of the wild type and odal were obtained in different nucleotide states: apo, AMP-PNP, ADP/ $V_{\mathrm{i}}$ and ADP. In the $\mathrm{ADP} / \mathrm{V}_{\mathrm{i}}$ state, the 24-nm meridional reflection of the wild type became over twice as strong as that in the apo state. Only in the $\mathrm{ADP} / \mathrm{V}_{\mathrm{i}}$ state, the $16-\mathrm{nm}$ equatorial reflection shifts away from the meridian, indicating compression of the axonemal lattice structure in the $\mathrm{ADP} / \mathrm{V}_{\mathrm{i}}$ state.

\section{$1 P 254$}

Conformations and chemical states of $F_{1}$-ATPase during rotation OTomoko Masaike', Masasuke Yoshida ${ }^{2}$, Kazuhiro Oiwa ${ }^{3}$, Takayuki Nishizaka' ${ }^{1}$ Dept. of Physics, Gakushuin Univ., ${ }^{2}$ Chemical Resources Lab., Tokyo Institute of Technology, ${ }^{3}$ National Institute of Information and Communication Technology

$\mathrm{F}_{0} \mathrm{~F}_{1}$-ATP synthase plays an essential role in living cells as a provider of universal source of energy, ATP. The $\alpha_{3} \beta_{3} \gamma$ subcomplex of $F_{1}$ part is its catalytic core which is able to hydrolyse ATP into ADP and inorganic phosphate at each catalytic site in the $\beta$ subunits. Crystal structures of $\alpha_{3} \beta_{3} \gamma$ by Abrahams et al. (Nature, 1994) and other bulk-phase experiments suggested that ATP hydrolysis reaction at the catalytic site triggers conformational changes between "Open" and "Closed" states of the C-terminal domain of the $\beta$ subunit and, as a result, drive rotation of the central stalk $\gamma$. Nevertheless, correlation between conformational changes and rotation has yet been unclear due to technical difficulties in concurrent detection of these two types of motion within the same molecule.

In order to visualise these causes and effects in powerstroke, simultaneous observation of conformational changes of $\beta$ and rotation of $\gamma$ in single $\alpha_{3} \beta_{3} \gamma$ molecules was performed under the microscope. A fluorescent molecule linked to the $\beta$ subunit was used as a probe of conformational changes. It was excited by an evanescent wave under total internal reflectance fluorescence microscopy. Meanwhile, rotation of polystyrene beads attached to $\gamma$ was observed under brightfield illumination.

In the meeting, we will present our recent findings from observation and discuss possible mechanisms of powerstroke from the viewpoint of conformational changes by incorporating conformation for each chemical state during rotational catalysis.

\section{$1 \mathrm{P} 256$}

Characterization of the Cys-introduced P-ring component FlgI and the stator component MotB in the flagellar motor of Escherichia coli

OYohei Hizukuri ${ }^{1}$, Seiji Kojima ${ }^{1}$, Ikuro Kawagishi ${ }^{1,2}$, Michio Homma ${ }^{1}$

'Div. of Biol. Sci., Grad. Sch. of Sci., Nagoya Univ., ${ }^{2}$ Inst. for Advanced Research, Nagoya Univ.

Bacterial flagellar rotation is driven by the motor embedded in the cytoplasmic membrane. Isolation of the flagellar basal body has already been established, but preparation of the functional flagellar motor has not been accomplished so far since the MotA/MotB stator complex is elusive during purification. To prevent the stator complex from detaching, we aimed at cross-linking between the P-ring protein FlgI and the stator component MotB. The P ring is located in the peptidoglycan layer, and a non-rotating component as a bushing to hold the rotating rod. On the other hand, the transmembrane protein MotB has a peptidoglycan binding motif in its $\mathrm{C}$ terminal periplasmic domain. To cross-link FlgI and MotB by a disulfide bond, we planned to generate 37 systematically-Cys-substituted FlgI variants and 13 MotB variants. Until now we have obtained $32 \mathrm{FlgI}$ and $13 \mathrm{MotB}$ Cys variants, and examined their function on motility. Almost all of the variants showed nearly wildtype functions, but expression of five of the FlgI variants and one of the MotB variants in corresponding mutant strains resulted in significantly impaired motility, and one FlgI mutant and one MotB mutant did not support motility. Immunoblotting of the Cys variants revealed that most of the FlgI variants showed a unique band-shift pattern under non-reducing condition. On the other hand, some of the MotB variants showed band shifts probably by dimer formation. Details of the series of the Cys mutants will be discussed. 